Teknokultura. Revista de Cultura Digital y Movimientos Sociales

ISSNe: $1549-2230$

https://dx.doi.org/10.5209/TEKN.69317

\title{
Campo, conexión y desigualdad. Hacia una economía política de las prácticas en la era del capitalismo digital
}

Mikel Barba del Horno ${ }^{1}$

Recibido: 7 de mayo de 2020 / Aceptado: 29 de junio de 2020 Open peer reviews

Resumen. En este artículo se presenta una reflexión sobre las potencialidades que, para el estudio de lo digital, presenta el marco teórico desarrollado por Pierre Bourdieu. Se parte de tres premisas epistemológicas: la existencia de una cierta continuidad estructural con la sociedad predigital, el rechazo del dualismo digital-analógico y una concepción del poder y la desigualdad que va más allá de lo puramente conectivo o informacional. A lo largo del texto se expone como la teoría de Bourdieu puede ser una herramienta potente a la hora de abordar algunos de los debates que se han planteado en el seno de la sociología digital: los debates en torno a la presencia de actores no humanos, el ensamblaje ciborg digital o el concepto de prosumidor. Se defiende también la potencialidad del concepto de capital cultural objetivado para integrar lo digital en el universo de las prácticas sociales. Este concepto da cabida a elementos como el cuerpo, el espacio, los datos o la infraestructura en la explicación de la desigualdad.

Palabras clave: Bourdieu; capital cultural; capital social; sociología digital.

\section{[en] Field, connection, and inequality. Towards a political economy of practices in the digital capitalism era}

\begin{abstract}
This article talks about the potentialities of Pierre Bourdieu's theoretical framework for the social research on the digital. We start from three epistemological assumptions: structural continuity with the pre-digital society, the rejection of digital-analogical dualism and a conception of power and inequality that goes beyond the purely connective or informational. Throughout the text, we claim that Bourdieu's theory can be a powerful tool for dealing with some of the central debates in digital sociology: the debates around non-human actors, cyborg-digital assemblage, or prosumer concept. We defend the potentiality of objectified cultural capital concept to integrate the digital in the universe of social practices. This concept accommodates elements like body, space, data, or infrastructure in the explanation of inequality.
\end{abstract}

Keywords: Bourdieu; cultural capital; digital sociology; social capital.

Sumario. 1. ¿Capitalismo digital o lo digital en el capitalismo? 2. Conexión, identidad y desigualdad ¿Por qué recuperar a Bourdieu? 3. La teoría de campos de Pierre Bourdieu. 4. Hacia una economía política de las prácticas digitales 5. Referencias

Cómo citar: Barba del Horno, M. (2020). Campo, conexión y desigualdad. Hacia una economía política de las prácticas en la era del capitalismo digital. Teknokultura. Revista de Cultura Digital y Movimientos Sociales, 17(2), 121-130.

\section{1. ¿Capitalismo digital o lo digital en el capitalismo?}

El concepto de capitalismo digital es confuso en la literatura sobre el tema y puede dar lugar a equívocos. Como ha planteado Pace (2018), el término ha sido utilizado en ocasiones desde un punto de vista puramente nominal, para denominar al capitalismo en la era de las tecnologías digitales. Otros autores han intentado sostener un concepto estructural según el cual la revolución digital implicaría la emergencia de una nueva estructura de funcionamiento del capitalismo. El problema aquí es que los impactos de las tecnologías digitales se produ- cen en muchos niveles diferentes y son objeto de luchas, por lo que es difícil definir una estructura acabada que resuma todos los fenómenos derivados de la denominada revolución digital.

En este trabajo se adopta una concepción del capitalismo digital que parte de tres principios: la continuidad con muchos de los elementos de la estructura social que existía antes de la emergencia de lo digital, una definición del poder y la desigualdad no fundamentada exclusivamente en términos de conexión y una concepción no dicotómica de relaciones sociales digitales y analógicas. 


\subsection{Revolución digital y continuidad analógica: cuando el 5G despertó el dinosaurio aún estaba allí}

Las dinámicas de la desigualdad en el contexto del capitalismo digital requieren un abordaje que otorgue una importancia sustancial a las prácticas ligadas a esta nueva dimensión digital sin olvidar que todo el nuevo arsenal de nuevas formas de comunicación, poder e información se despliega sobre una estructura social fuertemente asentada que, aunque parcialmente dinámica, incluso líquida (Bauman, 2015), presenta importantes elementos de continuidad y resistencia al cambio.

La literatura de lo digital ha tenido cierta tendencia a centrarse en lo nuevo olvidándose, en ocasiones, de lo viejo (Chadwick, 2017), a pecar, en ocasiones, de cierto determinismo tecnológico y cierto epocalismo (Savage, 2013). Pero muchos de los problemas que se presentan con nuevas características en el nuevo mundo digital son como el dinosaurio de Monterroso que ya se encontraban ahí antes del advenimiento de internet. Lo que ahora llamamos fake news no sería muy diferentes en el fondo de la propaganda de siempre que, tuvo un lugar central en el ascenso de la extrema derecha en otras épocas, pero cuya presencia también ha sido una constante en los regímenes liberales (Stanley, 2016). La supuesta fragmentación cultural originada por las llamadas filter bubbles (Pariser, 2011) no tiene parangón en sus consecuencias -al menos hasta ahora- con siglos de barreras económicas y culturales entre clases sociales, con la estricta educación basada en roles de género o con diseños urbanísticos basados en la segregación racial.

En el ámbito del trabajo también se ha visto a empresas como Amazon, a través de su proyecto del Turco Mecánico, Uber o Cabify como causantes de un deterioro de las condiciones laborales sin precedentes. Sin negar los efectos de estas nuevas plataformas en los procesos de desregulación del trabajo (OIT, 2019), no hay que olvidar que son procesos que vienen de lejos y que se han ido consolidando durante décadas a través de la globalización neoliberal, la deslocalización o la externalización de tareas que, aunque no tengan un carácter digital, no dejan de ser procesos tecnológicos, además de jurídicos, políticos y culturales.

Los determinismos tecnológicos tienden a describir una relación unidireccional entre tecnología y sociedad, por lo que acaban proponiendo soluciones muy conectadas a la supuesta esencia técnica de los problemas que pretenden arreglar y, de esta manera, lejos de transformar las lógicas de funcionamiento de la vieja estructura contribuyen a reproducirla (Morozov, 2013). Desde la economía colaborativa como solución a los excesos del capitalismo, al uso de algoritmos para combatir las fake news (Tschiatschek et al., 2018) se plantean soluciones técnicas a los que serían problemas políticos y sociales de amplio calado.

Frente a los que, desde cierto determinismo tecnológico, preveían unas consecuencias inevitables - positivas o negativas según la lectura- en las dinámicas sociales y políticas, cada vez más autores defienden que las consecuencias de la tecnología permanecen abiertas y son, en gran medida, imprevisibles (Rendueles y Sádaba, 2019).
Si partimos de que no existe una ruptura histórica, un abordaje teórico de las prácticas digitales no puede despreciar las herramientas legadas por siglos de teoría social. En muchos de los análisis del capitalismo digital está clara dicha continuidad con un intento de enriquecimiento de las viejas teorías al confrontarlas con las nuevas realidades. El análisis de la economía de las plataformas como un control monopolístico de recursos críticos (Khan, 2018; Rahman, 2018) muestra, bajo una nueva forma, un problema que ha sido una constante en la historia del capitalismo y que se ha planteado en numerosas ocasiones y desde diferentes perspectivas en las ciencias sociales.

Otro ejemplo de trabajo que no se deja llevar por el determinismo tecnológico y que conecta con las explicaciones sociales predigitales es el trabajo de Alexandra Rosenblat (2018) Uberland en el que la autora analiza las consecuencias del modelo laboral y comercial de Uber sobre los trabajadores desde una perspectiva que parte de un importante trabajo etnográfico y que integra el análisis de las consecuencias de la tecnología en un campo de dinámicas sociales más amplio que abarca el marco regulatorio, las alianzas con el cliente para conseguir poder político, las categorías de clase, raza, etc. En la misma línea irían trabajos como el de Noble (2018) en torno a los efectos reforzantes que motores de búsqueda como google tienen en el racismo o el de Eubanks (2018) que analiza los efectos que tiene sobre la desigualdad la aplicación de algoritmos en diferentes programas públicos.

\subsection{El relato frente al dato: el poder no es sólo conexión}

Otro de los excesos que se deben evitar a la hora de abordar una aproximación teórica a la desigualdad en el capitalismo digital es el de suponer que todas las dinámicas importantes del poder se articulan en torno al control de los datos y la información. Esta tendencia a pensar la conectividad y las redes sociales como la única o la principal dimensión en el estudio de lo social no es nueva y ya está presente en enfoques como la teoría de redes (Wellman et al., 1988) o las teorizaciones sobre la sociedad informacional de Manuel Castells (2004); pero ha sido objeto de una nueva vuelta de tuerca con el advenimiento del capitalismo digital (Lash, 2007). La teoría de la sociedad informacional ha sido criticada por presentar pretensiones totalizadoras, reducir la agencia humana al ámbito de la comunicación y plantear como vía principal para la mejora social la intensificación en el uso de las TIC (Lupač, 2018).

Si la teoría de redes plantea que las redes sociales son la fuente principal de distribución del poder en una estructura social, algunas teorías sobre Internet van un paso más allá y tienden a hacer una identificación determinista entre la forma de la infraestructura técnica de la red y la estructura social. De esta manera una internet centralizada daría lugar necesariamente a dinámicas sociales desigualitarias mientras que para fomentar la igualdad social sería pertinente promover una internet descentralizada. Estos planteamientos constituyen una fetichización extrema de la tecnología. 
Otros enfoques, sin llegar al determinismo de identificar arquitectura tecnológica y estructura social, otorgan un peso excesivo a la conectividad como elemento determinante del poder y la desigualdad. Aunque las teorías de la brecha digital han evolucionado desde unos planteamientos en los que se prestaba atención solamente a la posibilidad de acceso a la infraestructura material, hacia un concepto más rico que tiene en cuenta aspectos motivacionales, de destrezas y de uso (Van Deursen y Van Dijk, 2015), esta teoría sigue centrando el grueso de la explicación de la desigualdad en la variable de la conexión. Como ha planteado Lupač (2018), el concepto de brecha digital resulta insuficiente para dar una explicación satisfactoria de la desigualdad y su capacidad explicativa está ligada a elementos contextuales, de manera que mientras que en algunos casos una menor conexión da lugar a un menor poder en otros sucede exactamente lo contrario; que el que tiene poder no necesita estar conectado.

También se ha prestado atención a las prácticas de extracción y acumulación de datos por parte de algunas empresas. Estas prácticas otorgan un poder importante a gobiernos y compañías que tienen a su disposición cantidades ingentes de datos sobre ciudadanos y consumidores (Peirano, 2019). Indudablemente la disponibilidad de grandes cantidades de datos y la capacidad para procesarlos es una fuente importante de poder, por cuanto puede servir para tratar de prever la conducta de los individuos y para intentar manipularlos. Tendrá además efectos importantes en el desarrollo epistemológico de las propias ciencias sociales (Marres, 2017) que podrán articular aproximaciones cuantitativas a la realidad muchos más precisas, aunque no libres de sesgos.

Pero los procedimientos cuantitativos han mostrado continuamente sus limitaciones a la hora de realizar predicciones a futuro en ciencias sociales, y no solamente por una limitación en la cantidad o calidad de los datos. En las relaciones sociales se dan continuamente elementos contextuales que alteran los significados de cualquier variable que sea objeto de medición, por lo que los datos sociales cuantitativos siempre tienen un carácter artificial (Marres, 2017), son abstracciones creadas por el investigador. Si a esto le sumamos que, en ciencias sociales, el conocimiento altera la propia realidad que estudia, la pretendida capacidad del big data para prever los acontecimientos futuros parece limitada.

Sin negar que los datos y la información son un elemento importante, tampoco es conveniente reducir todas las dimensiones de la estructura social y las desigualdades a un plano informacional (Savage, 2013). Aunque algunos autores lo han puesto en duda (Lash, 2007), los relatos que se hacen sobre la realidad son una dimensión fundamental de la construcción de la legitimidad, el poder y la desigualdad. No es necesario partir, como hace Lash (2007), de una dicotomía estricta entre un relato hegemónico impuesto desde arriba y un poder inserto en las prácticas que emerge desde abajo. La visión del relato hegemónico impuesto desde arriba es una abstracción útil para entender la realidad; como también lo es el análisis de los relatos concretos que, en la práctica, aparecen fragmentados y son incorporados por los agentes de maneras diversas. El relato se manifiesta a través de las prácticas y las prácticas generan relatos. El trabajo de Bourdieu puede proporcionar una herramienta satisfactoria para afrontar este debate.

\subsection{Vínculo social digital versus analógico: una dicotomía inadecuada}

Otro de los procedimientos que conviene evitar es el de trazar una dicotomía estricta entre relaciones sociales digitales y analógicas (online y offline), lo que Nathan Ferguson (2011) denomina dualismo digital. Si nos referimos al vínculo social, la dicotomía digital-analógica es insostenible. Son más bien las interacciones las que pueden ser clasificadas en base a esa dicotomía. El vínculo social entre dos personas puede estar basado en interacciones a través de internet, teléfono, cara a cara, por carta o por una mezcla de todas ellas. Una interacción puntual puede dicotomizarse fácilmente; en el caso de una relación entre personas habría que recurrir a muchas arbitrariedades.

Incluso en este caso, una dicotomía de ese tipo puede ser poco adecuada atendiendo a la diversidad de interacciones en cada una de las categorías dicotómicas. Las interacciones a través de correo electrónico, por ejemplo, tienden a considerarse más formales y a presentar un menor contenido emocional que otro tipo de interacciones digitales, que podrían estar más cerca de una interacción cara a cara (Haste, 2005).

Se ha tratado también de contraponer la fragilidad, la liquidez de los vínculos establecidos de forma digital con la consistencia de los vínculos forjados cara a cara. Una buena parte de la investigación reciente tiende a relativizar esta idea. En una revisión de la literatura de los efectos psicológicos en los jóvenes, se concluye que no se puede establecer una relación unívoca entre la interacción digital y dimensiones como la sociabilidad, la empatía o el narcisismo y que muchos de los efectos dependen de elementos contextuales que requerirían investigación de tipo cualitativo para poder ser explicados adecuadamente (James et al., 2017). Otros estudios han destacado la importancia de las relaciones online para el surgimiento de vínculos relevantes en el funcionamiento de ciertas organizaciones criminales (Leukfeldt et al., 2017) o para la búsqueda de apoyo emocional por parte de madres adolescentes (Nolan et al., 2017).

Otro de los temas sociológicos tras la emergencia de lo digital alude a los posibles efectos desintegradores de las nuevas tecnologías sobre el vínculo social. Aquí tampoco se debería caer en el determinismo tecnológico. Como ha mostrado Jamieson (2013), los efectos del uso de dispositivos digitales en las relaciones familiares dependen en gran medida del contexto cultural y de la forma concreta en la que se usan dichos dispositivos. Mientras que en las familias asiáticas se da un uso colectivo de los dispositivos que refuerza las relaciones familiares, en Europa se ha observado un uso más individualizado y una tendencia al debilitamiento de los lazos familiares por el efecto de la tecnología.

Sería conveniente, por lo tanto, entender las dinámicas digitales y las analógicas como complementarias 
y mutuamente relacionadas (Chadwick, 2017). También resulta adecuado no presuponer ningún efecto a nivel general del uso de una tecnología determinada, sino determinar empíricamente los efectos de los usos sociales concretos de la tecnología.

\section{Conexión, identidad y desigualdad ¿Por qué recuperar a Bourdieu?}

Las nuevas tecnologías digitales han sido definidas como un hecho social total (Marres, 2017, p. 24), capaz de influir en multitud de dinámicas sociales. Se trata de tecnologías especialmente flexibles por cuanto se pueden implementar en cualquier actividad que implique intercambio de información; es decir, en casi la totalidad de las actividades humanas. Sin embargo, esto no debe llevarnos a un determinismo tecnológico en el que partiendo de la tecnología pretendamos explicar la configuración de la sociedad presente y futura. Precisamente el hecho de estar frente a un conjunto muy amplio de tecnologías, que además son muy flexibles en sus aplicaciones, provoca que sus eventuales consecuencias sociales sean más indeterminadas. Las tecnologías digitales, $\mathrm{y}$ las empresas que las implementan, pueden adaptarse y obtener beneficios en contextos democráticos y autoritarios, en economías basadas en el consumo masivo de combustibles fósiles o en otras orientadas a una transición hacia la sostenibilidad.

La investigación social de lo digital debería partir, por lo tanto, de las prácticas sociales y de cómo tecnologías concretas influyen en contextos sociales concretos. El análisis de lo digital se centraría así en los efectos concretos sobre un universo de prácticas.

La teoría de campos de Bourdieu ha sido reivindicada por autores como Mike Savage como una herramienta analítica adecuada para una aproximación a la estructura social que vaya más allá de los conceptos de red y conexión (Savage, 2013). El enfoque de Bourdieu es especialmente interesante porque, por un lado, parte de las prácticas, de la realidad empírica, y, por otro lado, integra dinámicas sociales que tienen que ver con la conexión, pero también con la creación de significados, las identidades, las instituciones, el conocimiento o el poder.

Ignatow y Robinson (2017) han hecho una revisión de la literatura que aplica herramientas derivadas del trabajo de Bourdieu al estudio de lo digital. Se repasan trabajos, como el de van Dijk (2015), que han postulado la emergencia de un nuevo tipo de capital informacional asociado a la revolución digital. La noción de capital informacional ha sido cuestionada por autores como Savage (2013) y es descartada en este trabajo por entender que los conceptos originales de Bourdieu tienen capacidad para integrar de manera satisfactoria las dinámicas digitales.

Otros trabajos analizan la brecha digital desde una perspectiva bourdiana muy conectada a la que se ha denominado tradición DiMaggio. Esta visión, que dominó la primera recepción del trabajo de Bourdieu en Estados Unidos, está muy centrada en la operacionalización cuantitativa del capital cultural, lo que acaba desconectando el concepto del resto del aparato teórico y especialmente de la noción de campo.

Ignatow y Robinson (2017) repasan también trabajos interesantes que conectan con una concepción más amplia del enfoque de Bourdieu cuyo máximo exponente es Lareau (2011). Estos trabajos indagan, por ejemplo, en como los diferentes habitus de clase orientan de maneras diferentes las prácticas digitales dando lugar a una mayor o menor obtención de capital en el campo. La reflexión sobre Bourdieu que se propone en este trabajo conecta más con esta última línea de análisis.

Otro artículo que ha hecho uso de los conceptos bourdianos para explicar la denominada tercera brecha digital es el de Calderón (2020), en el que se recurre al concepto de capital digital propuesto por Ragnedda (2018). El artículo muestra las retroalimentaciones que se producen entre capital económico, cultural y social por un lado y capital digital por otro, para una muestra de jóvenes nativos digitales. Se trata de un enfoque que tiene importantes puntos en común con el propuesto en este artículo y es sumamente interesante para explicar la tercera brecha digital, relacionada con las desigualdades derivadas del uso de la tecnología, de la incorporación a través de la interacción online de habilidades que pueden ser utilizadas offline. En este trabajo, sin embargo, se desecha el uso del concepto de capital digital por entenderse que las dimensiones digitales de la desigualdad pueden ser integradas en el esquema de Bourdieu sin la necesidad de recurrir a un concepto específico y que lo realmente relevante es distinguir entre las dimensiones materiales, incorporadas e institucionalizadas de lo cultural, más allá de que sean digitales $\mathrm{o}$ analógicas.

El trabajo de Bourdieu presenta tres virtudes importantes a la hora de aproximarse a lo digital. En primer lugar, Bourdieu entiende las prácticas insertas en una estructura social que, para su análisis, se descompone en diferentes campos sociales interconectados. La acotación de un campo se hace en base a un determinado tipo de capital, de poder, que se genera en el mismo y que está ligado a elementos culturales, jurídicos, burocráticos, contactos personales, etc. Aquí tenemos la primera ventaja; partir del campo como unidad de análisis permite acotar el objeto de la investigación y limitar el alcance de lo tecnológico como hecho social total a un espacio social concreto en el que se está generando un tipo específico de desigualdad; en el que se está luchando por un tipo de capital.

Otra de las ventajas de la teoría de campos es su capacidad para integrar lo digital como hecho social total que tiene impactos a muchos niveles. El enfoque de Bourdieu integra las dimensiones más estructurales con la acción, las dimensiones más macro como los discursos o el Estado y las más micro como la interacción. Nos va a permitir integrar en el análisis elementos como las interacciones, los discursos, el Estado, el cuerpo o el espacio. Todos estos temas han tenido un tratamiento importante en la sociología digital (Lupton, 2014) ya que, 
a pesar de que son elementos que preceden y trascienden el marco del capitalismo digital, el advenimiento de lo digital ha generado cambios importantes en la manera de entenderlos.

Por último, las herramientas teóricas de Bourdieu permiten integrar la dimensión de la conexión en el estudio de la estructura social, sin limitarse a definir la estructura en términos de conexiones. Las nociones de habitus y campo de Bourdieu incorporan al análisis de la estructura social elementos como la cultura, las identidades o el conocimiento experto; lo hacen además desde una perspectiva constructivista, anclada en las prácticas y, por tanto, muy pegada a los hechos empíricos, algo interesante en una época de cambio acelerado.

\section{La teoría de campos de Pierre Bourdieu}

El trabajo teórico de Bourdieu trata de integrar los enfoques centrados en la agencia y los enfoques estructurales (Ritzer, 2002) superando las que él consideraba una serie de falsas dicotomías: individual-colectivo, objetivosubjetivo, individuo-sociedad, interesado-desinteresado (Bourdieu, 1997).

En esta síntesis es central el concepto de habitus. El habitus es la estructura social incorporada por el individuo a lo largo de su trayectoria vital que, a pesar de ser única, está condicionada por la posición que ocupa en la estructura social. El habitus, como estructura incorporada, condiciona la acción de los individuos. Bourdieu define el habitus como "sistemas de disposiciones duraderas y transferibles, estructuras estructuradas predispuestas a funcionar como estructuras estructurantes" (Bourdieu, 2007, p. 86).

El habitus integra componentes cognitivos, actitudinales, normativos, culturales e identitarios que están presentes en el individuo y que son consecuencia de su posición social. Las personas que tiene una posición social similar presentan habitus similares.

Como apuntábamos anteriormente, Bourdieu concibe la estructura social como un todo dividido en diferentes campos. Los individuos y los grupos construyen campos sociales, espacios de significación, a través de los que tratan de convertir sus propios habitus en poder; en capital cultural en palabras de Bourdieu. Los diferentes campos funcionan de manera relativamente autónoma unos de otros. En cada uno de estos campos va a estar en juego un tipo diferente de capital cultural y se va a producir una lucha entre las personas que poseen el capital en cuestión y quieren mantenerlo y acumularlo, y aquellos que carecen del capital y buscan apropiarse de él (Bourdieu, 2005).

Bourdieu utiliza los conceptos de campo social y capital cultural para describir cómo las clases dominantes tratan de crear una estructura en la que sus pautas culturales -sus habitus- sean dominantes. La tenencia de capital cultural da acceso a otros recursos simbólicos y materiales que colocan a los que poseen este capital en una situación de ventaja respecto a los que carecen del mismo.
Además del capital cultural y el económico, en el campo operan otros dos capitales: el simbólico y el social. El capital simbólico se manifiesta cuando una desigualdad es legitimada y se convierte en fuente de estatus a través de un proceso que Bourdieu denomina violencia simbólica (Bourdieu, 1997).

El capital social, por su parte, es especialmente interesante en el estudio de las dinámicas sociales en la era digital porque está ligado a la conexión. Estaría constituido por todas aquellas conexiones con otros individuos o grupos que otorgan acceso a recursos (Bourdieu, 1986, p. 21). En torno al concepto de capital social existe una gran confusión terminológica (Portes, 1998). Mientras Bourdieu define el capital social como un recurso de los individuos derivado de la conectividad que otorga una ventaja a los individuos que lo poseen; el concepto utilizado por autores como Putnam (1995) lo caracteriza como un recurso colectivo, una especie de cohesión social que beneficia al conjunto de la sociedad. Como muestra Portes la visión de Putnam mezcla el capital social con las fuentes de las que se origina y con los efectos a los que da lugar.

Una de las críticas que se le ha hecho a la obra de Bourdieu es que presenta un sesgo dominocéntrico (Grignon y Passeron, 1992). En su esquema pareciera que las prácticas de las clases dominadas acaban orientándose únicamente a reproducir su dominación. Esto es, en parte, consecuencia de que Bourdieu centró gran parte de su obra académica en la explicación de la reproducción de las desigualdades de clase; pero el concepto de campo es flexible y puede ser utilizado también para dar cuenta de las dinámicas de impugnación.

\section{Hacia una economía política de las prácticas digitales}

El proyecto de Bourdieu se podría resumir como un intento de aplicar la lógica del análisis económico marxista a todo el universo de las prácticas sociales; en este sentido, propone una ciencia general de la economía de las prácticas, que intente "aprehender el capital y el beneficio en todas sus formas, y determinar los procesos a través de los cuales el capital (o de poder, que es en definitiva lo mismo) pasa de una forma a otra" (Bourdieu, 1988, p. 16). Estudiar las prácticas relacionadas con lo digital desde la perspectiva de la teoría de campos presenta, como veremos, cierto potencial para articular algunos de los debates que han surgido en torno a la sociología digital.

Para definir el papel que tiene lo digital en los campos sociales conviene partir de la distinción entre datos y conocimiento. Los datos tienen un soporte material y pueden ser acumulados pero lo que realmente otorga una ventaja en el campo social es la capacidad de procesar dichos datos y convertirlos en conocimiento, es decir incorporarlos al habitus, para que luego puedan ser la base de alguna forma de institucionalización que genere desigualdad o ventaja para el grupo que la articula. Es importante destacar que, a partir de los datos, se pueden instituir formas de desigualdad, pero que éste no es un 
proceso automático, aunque pueda estar en parte automatizado (Eubanks, 2018), sino un proceso social que requiere siempre acción humana y que puede ser objeto de impugnación.

\subsection{La infraestructura digital como capital cultural objetivado}

Lo material ha adquirido una importancia central en torno a las teorizaciones sobre la sociedad digital. Así, conceptos como plataforma, archivos, infraestructura, dispositivos o ensamblaje digital han proliferado en los últimos años. Subyace la idea de una especie de materialización de las interacciones sociales mediadas por la tecnología, que pasan a ser codificadas en datos, almacenadas en diferentes archivos y mercantilizadas.

Las dinámicas económicas, políticas, técnicas y legales derivadas de la emergencia de esta nueva materialidad mercantilizada han sido estudiadas en multitud de trabajos (Jiménez González, 2020). Aunque estas dinámicas podrían ser analizadas a través de un campo específicamente digital, centrado en explicar el despliegue jurídico o empresarial de esta industria digital; lo que se propone a continuación es una forma de integrar la dimensión de lo digital en los campos sociales no específicamente digitales.

Una posible manera de integrar la infraestructura digital en la teoría de campos es a través del concepto de capital cultural objetivado. Bourdieu (1979) propone tres formas en las que se manifiesta el capital cultural: la incorporada, la institucionalizada y la objetivada. La primera remite al habitus, la segunda a las credenciales académicas que dan consistencia legal a ese habitus y la objetivada son aquellos elementos materiales, libros por ejemplo, que pueden ser transmitidos físicamente y que facilitan la adquisición incorporada del capital cultural. Es esta tercera forma de capital cultural la que nos interesa a la hora de introducir en el análisis del campo la infraestructura digital.

El capital objetivado se refiere a los elementos materiales, acumulables que dan acceso a nuevos repertorios culturales y que pueden proporcionar capital cultural incorporado. Bourdieu pone el ejemplo de los libros o las obras de arte. El capital cultural objetivado es fácilmente transmisible y no está indefectiblemente unido a la persona como el capital incorporado; sin embargo, la capacidad de convertir ese capital cultural objetivado en capital incorporado sí que depende de la persona que lo utiliza.

Desde este punto de vista, los datos pueden ser considerados una forma de capital cultural objetivado. Pueden ser fácilmente transferidos, comprados o vendidos y utilizados para convertirlos en conocimiento útil para las luchas en el campo, dando lugar a la acumulación de capital cultural incorporado.

Bennet y otros (Bennett et al., 2008) han defendido que el capital cultural objetivado está también conectado al capital social. Estos autores sostienen que el acceso a espacios como la ópera constituye una forma de capital cultural objetivado que proporciona conexiones, es decir, capital social.

Si algo pone de manifiesto la emergencia de lo digital es la importancia de tener acceso a la conexión con los demás, y la clara posibilidad de apropiación privada de las infraestructuras que permiten esa conexión; o lo que es lo mismo una creciente relación entre capital económico y capital social. En este sentido, podría plantearse que el capital cultural objetivado son también aquellos elementos materiales que permiten las conexiones con otros. Dentro de esta categoría entrarían elementos asociados al cuerpo y al espacio.

Las interacciones con otras personas se realizan a través del cuerpo y el espacio. Para que se dé una interacción cara a cara, los cuerpos tienen que coincidir en el espacio físico. Existen elementos del cuerpo que pueden favorecer o limitar el acceso a determinados espacios. Algunos son modificables y otros tienen una base genética. La discriminación que se ejerce sobre determinados fenotipos, o sobre determinados signos corporales, vestimentas o peinados limita el acceso a espacios de socialización y fomenta la segregación espacial; restringiendo el capital social y, de esta manera, las posibilidades de modificar el habitus a través de la aculturación para obtener capital cultural. Los espacios también pueden ser objeto de apropiación privada y pueden tener el acceso restringido. Vemos pues que existen elementos del cuerpo y del espacio que limitan el acceso a ciertos lugares del campo y que son apropiables a través del capital económico.

En la era digital las interacciones pueden tener lugar, además de en el espacio físico, en el espacio virtual. Este espacio está constituido por un conjunto de dispositivos tecnológicos que son apropiables económicamente y que generan cantidades enormes de datos que se encuentran, en principio, bajo el control de los propietarios de dichos dispositivos. Por lo tanto, el acceso al espacio virtual requiere pagar por utilizar los dispositivos que lo integran, ceder los datos generados en la interacción al propietario de las tecnologías o ambas.

En cuanto al cuerpo en el espacio virtual, algunos autores han planteado que nos encontramos, cada vez más, ante cuerpos que mezclan características biológicas y tecnológicas. En este sentido ha trabajado Deborah Lupton, inspirándose en los ciborg de Haraway, para desarrollar el concepto de ensamblaje ciborg digital, que se refiere al cuerpo aumentado por el uso de una serie de tecnología digitales que se insertan en el mismo cuerpo e interactúan con él (Lupton, 2014, p. 165). Se ha producido una penetración de la tecnología en el cuerpo y una extensión del cuerpo a través de la tecnología. Para acceder al espacio virtual es necesario conectar el cuerpo biológico a una serie de extensiones tecnológicas que también son objeto de apropiación económica, son también, en este sentido, capital cultural objetivado. Tenemos pues que tanto los dispositivos digitales como los datos o la infraestructura digital encajan en la teoría de campos a través del concepto de capital cultural objetivado. 
Figura 1. Cuerpo y espacio en el campo social. Fuente: elaboración propia

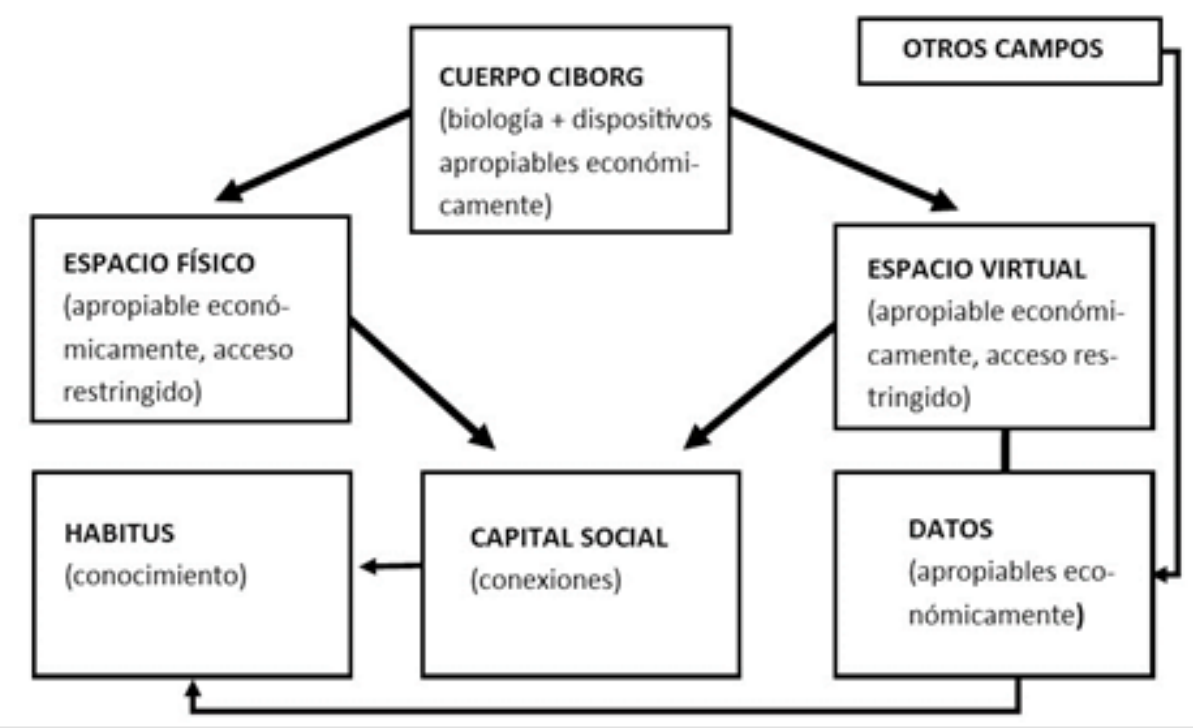

Las relaciones entre cuerpo y espacio se producirían como muestra la figura 1. Tenemos que tanto el espacio físico como el digital son apropiables a través del capital económico. Lo mismo ocurre con los dispositivos tecnológicos a los que hay que conectar el cuerpo físico para acceder al espacio digital. Todas las interacciones que se producen en el espacio digital generan además datos que pueden ser vendidos y pueden ser empleados en el campo en el que se generan, o en otros campos, para obtener conocimiento. Los procesos que aparecen la figura 1 pueden, además, ser objeto de regulación por parte del Estado, por lo que su funcionamiento concreto dependerá del resultado de las luchas que se dan en el campo como se verá más adelante.

\subsection{Prácticas digitales (y analógicas) en el campo}

Las personas conforman sus habitus a través de sus trayectorias vitales, relacionándose con otras personas e intercambiando contenido cultural, información. Como hemos visto en el apartado anterior, las interacciones con otros pueden darse a través del espacio digital o del espacio físico. Los contactos que mantiene una persona con otras son capaces de generar acceso a recursos y constituyen lo que Bourdieu denomina capital social. Este capital social puede fundamentarse en relaciones cara a cara, en relaciones online o en una mezcla de ambas. La novedad en el capitalismo digital es que las interacciones digitales mediadas por dispositivos generan datos que son susceptibles de ser utilizados en el campo por los propietarios de dichos datos o por los que tengan el capital económico suficiente para adquirirlos. Esto, indudablemente puede tener efectos sobre las luchas que se producen en el campo.

En un campo social, los diferentes grupos, basados en la clase, la categoría profesional, la etnia, el género $\mathrm{u}$ otros, cuyos miembros presentan habitus similares, luchan por la creación y apropiación de capital. Se produce una lucha por definir las reglas del campo en beneficio del propio grupo, por generar desigualdades y por lograr que esas desigualdades sean percibidas como legítimas. Los grupos articulan discursos, generan iden- tidades grupales y marcos interpretativos de la realidad que los lleven a obtener ventajas sobre los otros grupos. Como apuntábamos anteriormente las conexiones, el capital social, son importantes en el campo, pero también lo son los discursos, los procesos de institucionalización y estatalización, la formación de grupos con unos valores comunes, las identidades, etc.

En todas estas dinámicas el conocimiento tiene un papel fundamental. Los datos que se generan en las interacciones pueden ser utilizados y convertidos en conocimiento por parte de ciertos grupos que tengan acceso a los mismos. Esto puede otorgarle una ventaja a la hora de definir el campo en beneficio propio. Los datos pueden ser empleados de varias maneras: para tener un conocimiento más preciso de aquel a quien se quiere dominar; pero también, y esto es incluso más importante, pueden servir para instituir como verdad oficial determinadas formas de conocimiento, y así hacer pasar por universal un punto de vista que en realidad es particular (Bourdieu, 2014). Volveremos sobre este punto al hablar del papel del Estado en el campo.

El papel que tienen los algoritmos como elementos mediadores en el procesamiento de datos (Besteman y Gusterson, 2019) está también vinculado al conocimiento experto y presenta caracteres polimórficos según el uso concreto que se le dé. Su manifestación en el campo presenta similitudes, según el caso, con diferentes tecnologías sociales como los procedimientos organizativos burocráticos, la praxis científica, prácticas discriminatorias normativizadas o tecnologías de vigilancia.

Los conceptos de prácticas y habitus puede servir también para situar el debate en torno al ensamblaje humano-algoritmo (Latour, 2005), que ha llegado a plantear la caracterización de los algoritmos y las máquinas como actores no humanos. Si partimos de que las prácticas son fruto de la acción humana, pero también de la estructura incorporada, del habitus; se relativiza la necesidad de incluir en la explicación actores no humanos. Desde este punto de vista, los algoritmos, al igual que el habitus, contribuyen a automatizar las prácticas humanas, a orientar la acción; pero forman parte de la estructura. Lo que caracteriza la acción humana es, pre- 
cisamente, la libertad en potencia, la capacidad para escapar, siquiera de forma limitada, a esos condicionantes estructurales.

El argumento de que los algoritmos funcionan como una caja negra (Pasquale, 2015) que esconde los mecanismos de poder haciéndolos invisibles, se puede aplicar a la mayoría de las dinámicas de legitimación racionalburocráticas que planteaba Weber, con la desventaja de que la legitimación a través de algoritmos, al no estar sostenida en un relato explícito es más incompleta. Los mecanismos de discriminación que ejecutan los algoritmos pueden ser desvelados por sus efectos; de hecho, a tenor de las críticas que suscitan, podría decirse que están siendo objeto de una impugnación muy temprana. Como muestra Eubanks (2018), las víctimas de los algoritmos son en ocasiones los primeros en percibir los efectos discriminatorios de los mismos.

El enfoque de campos es útil también para afrontar el debate surgido en torno al concepto de prosumidor. Situar el problema de la producción y mercantilización de datos en un plano que va más allá de lo estrictamente económico o que parte de una concepción incrustada de lo económico (Polanyi, 1957), permite dar una explicación más satisfactoria de la agencia de los usuarios de las plataformas digitales. El concepto de prosumidor (Fuchs y Dyer-Witheford, 2013) se ha mostrado útil para explicar cómo las plataformas extraen valor de lo que podría considerarse trabajo de producción de datos de los usuarios. Sin embargo, presenta limitaciones a la hora de abordar una práctica como el uso de plataformas digitales que puede tener matices, motivaciones y consecuencias muy diferentes según el contexto de cada campo social. Todo uso de una plataforma es susceptible de generar datos; pero también puede ser la fuente de un capital social útil en el campo. La teoría de campos nos ofrece otra perspectiva más centrada en las dinámicas sociales en las que se usa la tecnología que en la tecnología en sí.

Por último, la teoría de campos también contempla la inclusión de las dinámicas estatales. Esto es especialmente importante por cuanto el uso de las nuevas tecnologías digitales genera problemas relativos a la privacidad y a la regulación en el almacenamiento y uso de los datos. Bourdieu entiende el Estado de una manera difusa, no como una organización o conjunto de organizaciones sino como una serie de prácticas que tienden a cristalizar y a adquirir el carácter de normas dotadas de oficialidad y que acaban siendo percibidas como si fueran universales (Bourdieu, 2014). Los grupos tratan, a través de la obtención del reconocimiento de lo "estatal", de instituir permanentemente una ventaja que han obtenido en el campo social.

En este sentido, en un campo algunos actores podrían buscar el reconocimiento legal de los procesos de producción y uso de datos mientras que otros actores serían partidarios de la limitación de establecer límites legales restrictivos. Decíamos anteriormente que, en ocasiones, las dinámicas de poder que rodean los datos se perciben por sus consecuencias; es decir, se perciben en los campos en los que dichos datos son usados para discriminar o excluir a personas y colectivos. La impugnación de estas dinámicas es más probable cuando existe una consecuencia directa y perceptible del uso de los datos, que en un nivel de regulación general (Ozer, 2012). También podría resultar un enfoque adecuado para abordar las estrategias de legitimación que las plataformas ponen en marcha aliándose con los usuarios (Culpepper y Thelen, 2019) o con instancias científicas y estatales, como ejemplifica el caso de la epidemia de COVID-19 (Ting et al., 2020). Desde el Estado también se regula la posibilidad de acceso, restricción y privatización de los espacios físicos y virtuales, de manera que el funcionamiento concreto de la dinámica que se ilustraba en la figura 1 depende de las luchas en el campo y de la capacidad que los grupos tengan para institucionalizar -podríamos decir "estatalizar"- sus capitales.

Resumiendo, la teoría de campos de Bourdieu puede ser una herramienta interesante para integrar lo digital en el estudio de la estructura social y las desigualdades. Permite un análisis de la tecnología inserta en las prácticas concretas, tomando así distancia con los determinismos tecnológicos y con los epocalismos rupturistas. Además, otorga una importancia central a la conexión, a las redes y a la información sin descartar otras dimensiones de la desigualdad social. La distinción entre datos como capital objetivado y conocimiento como capital incorporado permite analizar en el contexto empírico las formas a través de las que los datos pueden convertirse en alguna forma de poder y desigualdad, superando de esta manera las visiones totalizantes que identifican de manera automática información y poder. La teoría de campos puede resultar una herramienta útil para afrontar de manera coherente muchos de los debates que se han planteado en la sociología digital. El análisis de los efectos sociales de los algoritmos puede ser enfocado desde el punto de vista empírico de un campo social concreto, indagando en cómo determinados actores ponen en juego los algoritmos para gestionar datos y convertirlos en un conocimiento incorporado que les permita situarse en una posición dominante, y cómo otros actores articulan sus prácticas parar impugnar el uso o los efectos de dichos algoritmos. En el debate sobre los ensamblajes humanos digitales, los conceptos de capital objetivado e incorporado pueden servirnos para explicar cómo se articulan los dispositivos, el cuerpo y los espacios virtual y analógico en las dinámicas de creación y apropiación de conocimiento en diferentes contextos sociales. Por último, el concepto de capital institucionalizado es útil para aproximarnos a las dinámicas legales en torno a los datos. La búsqueda por parte de las grandes compañías de un reconocimiento legal de la extracción y uso de los datos y las prácticas de impugnación que surgen contra dichos usos son más fácilmente analizables en el contexto de un campo social concreto que en un nivel general. 


\section{Referencias}

Bauman, Z. (2015). Modernidad líquida. Fondo de Cultura Económica.

Bennett, T., Savage, M., Silva, E. B., Warde, A., Gayo-Cal, M. y Wright, D. (2008). Culture, Class, Distinction. Taylor \& Francis. Besteman, C. y Gusterson, H. (2019). Life by Algorithms: How Roboprocesses Are Remaking Our World. University of Chicago Press.

Bourdieu, P. (1986). The forms of capital. En Handbook of Theory and Research for the Sociology of Education (J.G. Richardson, pp. 241-258). New York: Greenwood Press.

Bourdieu, P. (1979). Los tres estados del capital cultural. Sociológica, 5, 11-17.

Bourdieu, P. (1988). La distinción: criterios y bases sociales del gusto. Madrid: Taurus.

Bourdieu, P. (1997). Razones prácticas. Sobre la teoría de la acción. Barcelona: Anagrama.

Bourdieu, P. (2005). Una invitación a la sociología reflexiva (1a ed.). Argentina: Siglo XXI Editores.

Bourdieu, P. (2007). El sentido práctico. Argentina: Siglo XXI Editores.

Bourdieu, P. (2014). Sobre el Estado: cursos en el Collège de France (1989-1992). Barcelona: Anagrama.

Calderón Gómez, D. (2020). The third digital divide and Bourdieu: bidirectional conversion of economic, cultural, and social capital to (and from) digital capital among young people in Madrid. New Media \& Society. https://doi.org/10.1177/1461444820933252.

Castells, M. (2004). La era de la información: Economía, Sociedad y Cultura. Argentina: Siglo XXI Editores.

Chadwick, A. (2017). The Hybrid Media System: Politics and Power. Oxford: University Press.

Culpepper, P. D. y Thelen, K. (2019). Are We All Amazon Primed? Consumers and the Politics of Platform Power: Comparative Political Studies. https://doi.org/10.1177/0010414019852687.

Eubanks, V. (2018). Automating Inequality: How High-Tech Tools Profile, Police, and Punish the Poor. New York: St. Martin's Publishing Group.

Ferguson, N. (2011). Digital Dualism versus Augmented Reality. Cyborgology. Recuperado de https://thesocietypages.org/ cyborgology/2011/02/24/digital-dualism-versus-augmented-reality/.

Fuchs, C. y Dyer-Witheford, N. (2013). Karl Marx @ Internet Studies. New Media y Society, 15(5), 782-796. https://doi. org/10.1177/1461444812462854.

Grignon, C. y Passeron, J.C. (1992). Lo culto y lo popular: miserabilismo y populismo en sociología y en literatura. Madrid: Ediciones La Piqueta.

Haste, H. (2005). Joined-Up Texting: The role of mobile phones in young people's lives. London: Nestlé Social Research Programme.

Ignatow, G. y Robinson, L. (2017). Pierre Bourdieu: Theorizing the digital. Information, Communication y Society, 20(7), 950966. https://doi.org/10.1080/1369118X.2017.1301519.

James, C., Davis, K., Charmaraman, L., Konrath, S., Slovak, P., Weinstein, E., y Yarosh, L. (2017). Digital Life and Youth Wellbeing, Social Connectedness, Empathy, and Narcissism. Pediatrics, 140 (Supplement 2), S71-S75. https://doi.org/10.1542/ peds.2016-1758F.

Jamieson, L. (2013). Personal Relationships, Intimacy and the Self in a Mediated and Global Digital Age. En Digital Sociology: Critical Perspectives (K. Orton-Johnson y N. Prior, pp. 13-33). Palgrave Macmillan UK.

Jiménez González, A. (2020). Tech power: a critical approach to digital corporations. Teknokultura, 17(1), 77-85. https://doi. org/10.5209/tekn.66931.

Khan, L. (2018). Sources of Tech Platform Power. Social Science Research Network. Recuperado de https://papers.ssrn.com/ abstract $=3558741$.

Lash, S. (2007). Power after Hegemony: Cultural Studies in Mutation? Theory, Culture y Society, 24(3), 55-78. https://doi. org/10.1177/0263276407075956.

Latour, B. (2005). Reassembling the Social: An Introduction to Actor-Network-Theory. Oxford: Oxford University Press.

Leukfeldt, E. R., Kleemans, E. R. y Stol, W. P. (2017). Cybercriminal Networks, Social Ties and Online Forums: Social Ties Versus Digital Ties Within Phishing and Malware Networks. The British Journal of Criminology, 57(3), 704-722. https://doi. org/10.1093/bjc/azw009.

Lupač, P. (2018). Beyond the Digital Divide: Contextualizing the Information Society. Reino Unido: Emerald Group Publishing.

Lupton, D. (2014). Digital Sociology. New York: Routledge.

Marres, N. (2017). Digital Sociology: The Reinvention of Social Research. Londres: John Wiley \& Sons.

Morozov, E. (2013). To Save Everything, Click Here: The Folly of Technological Solutionism. London: PublicAffairs.

Noble, S. U. (2018). Algorithms of Oppression: How Search Engines Reinforce Racism. New York: NYU Press.

Nolan, S., Hendricks, J., Ferguson, S., y Towell, A. (2017). Social networking site (SNS) use by adolescent mothers: Can social support and social capital be enhanced by online social networks? - A structured review of the literature. Midwifery, 48, 2431. https://doi.org/10.1016/j.midw.2017.03.002.

OIT. (2019). Las plataformas digitales y el futuro del trabajo: Cómo fomentar el trabajo decente en el mundo digital. Organización Internacional del Trabajo. Recuperado de https:/www.ilo.org/global/publications/books/WCMS_684183/lang--es/index.htm.

Ozer, N. A. (2012). Putting Online Privacy above the Fold: Building a Social Movement and Creating Corporate Change. New York University Review of Law \& Social Change, 36, 215.

Pace, J. (2018). The Concept of Digital Capitalism. Communication Theory, 28(3), 254-269. https://doi.org/10.1093/ct/qtx009

Pariser, E. (2011). The Filter Bubble: How the New Personalized Web Is Changing What We Read and How We Think. New York: Penguin Random House.

Pasquale, F. (2015). The Black Box Society. New York: Harvard University Press.

Peirano, M. (2019). El enemigo conoce el sistema: Manipulación de ideas, personas e influencias después de la economía de la atención. España_Penguin Random House.

Polanyi, K. (1957). El sistema económico como proceso institucionalizado. En Entre las gracias y el molino satánico. Lecturas de Antropología Económica.(P. Moreno, pp. 155-1789. Madrid: UNED Editorial. 
Portes, A. (1998). Social Capital: Its Origins and Applications in Modern Sociology. Annual Review of Sociology, 24(1), 1-24.

Putnam, R. D. (1995). Bowling Alone: America's Declining Social Capital. Journal of Democracy, 6(1), 65-78. https://doi. org/10.1353/jod.1995.0002.

Ragnedda, M. (2018). Conceptualizing Digital Capital. Telematics and Informatics, 35(8), 2366-2375. https://doi.org/10.1016/j. tele.2018.10.006.

Rahman, K. S. (2018). Infrastructural Regulation and the New Utilities (SSRN Scholarly Paper ID 3205994). Social Science Research Network. Recuperado de https://papers.ssrn.com/abstract=3205994.

Rendueles, C., y Sádaba, I. (2019). Digitalización y cambio social: De las expectativas apocalípticas a la tecnopolítica del presente. Cuadernos de relaciones laborales, 37(2), 331-349. https://doi.org/10.5209/crla.66041.

Ritzer, G. (2002). Teoría sociológica moderna (5a ed.). New York: MacGraw-Hill.

Rosenblat, A. (2018). Uberland: How Algorithms Are Rewriting the Rules of Work. California: Univ of California Press.

Savage, M. (2013). Digital Fields, Networks and Capital: Sociology beyond Structures and Fluids. En Digital Sociology: Critical Perspectives (K. Orton-Johnson y N. Prior, pp. 139-147). Reino Unido: Palgrave Macmillan.

Stanley, J. (2016). How Propaganda Works. Nueva Jersey: Princeton University Press.

Ting, D. S. W., Carin, L., Dzau, V. y Wong, T. Y. (2020). Digital technology and COVID-19. Nature Medicine, $26(4), 459-461$. https://doi.org/10.1038/s41591-020-0824-5.

Tschiatschek, S., Singla, A., Rodríguez, M., Merchant, A. y Krause, A. (2018). Fake News Detection in Social Networks via Crowd Signals. 517-524. https://doi.org/10.1145/3184558.3188722.

Deursen, A. J. A. M. van y Dijk, J. A. G. M. van. (2015). Toward a Multifaceted Model of Internet Access for Understanding Digital Divides: An Empirical Investigation. The Information Society, 31(5), 379-391. https://doi.org/10.1080/01972243.20 15.1069770.

Wellman, B., Berkowitz, S. D., y Granovetter, M. (1988). Social Structures: A Network Approach. Cambridge: CUP Archive. 